\title{
Gap Solitons in Anharmonic Diatomic lattice
}

\author{
Nand Kishore Pandey ${ }^{1}$, Gautam Johri ${ }^{2}$, Anil Kumar Ram ${ }^{3}$ \\ Department of Postgraduate Studies and Research in Physics and Electronics, Rani Durgavati University Jabalpur-482001, India
}

\begin{abstract}
We consider a one -dimensional nonlinear diatomic lattice with two different masses (M>m). We find the exact soliton solutions in the forbidden frequency range (band gap) between the longitudinal acoustic and optical phonon branches by using coupled wave method.
\end{abstract}

Keywords: Double Sine -Gordon Equation, Critical points, Linear stability analysis, Saddle point, Gap-Solitons, Coupled wave method, Forbidden frequency gap

\section{Introduction}

Physically, the natures of nearest neighbour interaction in materials are nonlinear. These gives rise to nonlinear localized modes in forbidden region in the linear phonon spectrum .We investigate such localized modes near a Bragg resonance of a nonlinear diatomic chain by the analytical methods. Earlier works to investigate these modes have been done by numerical method $[1,2]$. The analytical solutions found by the method of Green's function are too cumbersome and difficult to analyze regarding the question of the different mechanism of the nonlinear brightening of the diatomic lattice. We study this problem by using the coupled wave method[3, 4] .In this method the solution of the wave equation for a one dimensional periodic medium is represented as the superposition of two opposing waves with amplitudes varying slowly with position. The problem thus reduce to a system of a coupled first order equation which can include dynamic characteristics of the medium[5,6] .Under certain conditions, the lattice becomes transparent at the margins of the forbidden gap between longitudinal acoustic and optical phonons, due to self-action of the wave. We investigate the occurrence of these gap solitons in the region where no linear modes exists in the diatomic lattice[7, $8,9,10,11]$.

\section{Model}

We consider the nonlinear interaction potential between the nearest neighbours to be of the quartic type. Therefore the equation of the motion for both types of atoms can be written as

$$
\begin{array}{r}
\mathrm{m}_{0}(1+\eta \cos \pi) \ddot{X}_{\mathrm{n}}=\mathrm{K}_{2}\left[\mathrm{X}_{\mathrm{n}+1}+\mathrm{X}_{\mathrm{n}-1}-2 \mathrm{x}_{\mathrm{n}}\right]+\mathrm{K}_{4} \\
{\left[\left(\mathrm{X}_{\mathrm{n}+1}-\mathrm{X}_{\mathrm{n}}\right)^{3}-\left(\mathrm{X}_{\mathrm{n}}-\mathrm{X}_{\mathrm{n}-1}\right)^{3}\right]-(1)}
\end{array}
$$

When for ' $n$ ' even the equation describe the motion of heavier atoms and for ' $n$ ' odd that of lighter atoms. In the linear case, the wave propagation of certain wave length is forbidden (solution do not exist between $\sqrt{ } 2 \omega_{1}$ and $\sqrt{ } 2 \omega_{2}$, Where $\omega_{1}^{2}=\frac{K_{2}}{M}$ and $\omega_{2}^{2}=\frac{K_{2}}{m}$ ). Gap solitons, are a result of the balance between week nonlinearity and dispersion [12].

In the frequency falls into the forbidden region, then the strong coupling arises between the waves, so we can represent the solution in the form of a pair oppositely propagating waves with amplitudes of waves with close than phase velocities. This is called the coupled wave method $[3,4]$. The solution can be written as

$$
\begin{array}{r}
X_{n}=A_{1}(n) \exp \left[i\left(\omega t-n \frac{\pi}{2}\right)\right]+A_{2}(n) \exp \left[i\left(\omega t+\frac{n \pi}{2}\right)\right]+ \\
A_{1}^{*} \exp \left[-\omega t+\frac{i n \pi}{2}\right]+A_{2}^{*}(n) \exp \left[-i \omega t-\frac{i n \pi}{2}\right]-(2)
\end{array}
$$

Equation(1) andequation(2) can be solved to obtain the amplitude of vibration of both types of atoms of the diatomic lattice in the forbidden region of the linear spectrum $\left(2 \sqrt{\omega_{1}}<\omega<2 \sqrt{\omega_{2}}\right)$.

\section{Gap Soliton solution}

The problem then reduces to a system of coupled first order equations. From expression (2)substituting in equation (1)then we obtain

$$
\begin{gathered}
\frac{\mathrm{i}}{2}\left[\mathrm{~A}_{1}(\mathrm{n}+1)-\mathrm{A}_{1}(\mathrm{n}-1)\right]+\Delta \mathrm{A}_{1}(\mathrm{n})-\sigma \mathrm{A}_{2}(\mathrm{n})+\chi_{12}(\mathrm{n}) \\
=0-(3-a) \\
\begin{aligned}
\frac{\mathrm{i}}{2}\left[\mathrm{~A}_{2}(\mathrm{n}-1)-\mathrm{A}_{2}(\mathrm{n}+1)\right]+\Delta \mathrm{A}_{2}(\mathrm{n})-\sigma \mathrm{A}_{1}(\mathrm{n})+\chi_{21}(\mathrm{n}) \\
=0-(3-\mathrm{b})
\end{aligned}
\end{gathered}
$$

Where $\sigma=\frac{\mathrm{m}_{0} \eta \omega^{2}}{2 \mathrm{~K}_{2}}$ and $\Delta=1-\frac{\omega^{2} \mathrm{~m}_{0}}{2 \mathrm{~K}_{2}}$ and

$$
\begin{aligned}
\chi_{12}=\frac{\chi}{2}\left[\mathrm{~A}_{1}(\mathrm{n})\{\mid\right. & \left.\mathrm{A}_{1}(\mathrm{n})\right|^{2}+2\left|\mathrm{~A}_{2}(\mathrm{n})\right|^{2}+\left|\mathrm{A}_{1}(\mathrm{n}-1)\right|^{2} \\
& +\left|\mathrm{A}_{2}(\mathrm{n}-1)\right|^{2}+\left|\mathrm{A}_{1}(\mathrm{n}+1)\right|^{2} \\
& \left.+\left|\mathrm{A}_{2}(\mathrm{n}+1)\right|^{2}\right\}+\frac{1}{2} \mathrm{~A}_{1}^{*}(\mathrm{n})\left\{2 \mathrm{~A}_{2}^{2}(\mathrm{n})\right. \\
& -\mathrm{A}_{1}^{2}(\mathrm{n}-1)-\mathrm{A}_{2}^{2}(\mathrm{n}-1)-\mathrm{A}_{1}^{2}(\mathrm{n}+1) \\
& \left.-\mathrm{A}_{2}^{2}(\mathrm{n}+1)\right\}+\mathrm{A}_{1}(\mathrm{n} \\
& -1)\left\{\mathrm{A}_{2}^{*}(\mathrm{n}) \mathrm{A}_{2}(\mathrm{n}-1)-\mathrm{A}_{2}(\mathrm{n}) \mathrm{A}_{2}^{*}(\mathrm{n}\right. \\
& -1)\}+\mathrm{A}_{1}(\mathrm{n}+1)\left\{\mathrm{A}_{2}^{*}(\mathrm{n}) \mathrm{A}_{2}(\mathrm{n}+1)\right. \\
& \left.-\mathrm{A}_{2}(\mathrm{n}) \mathrm{A}_{2}^{*}(\mathrm{n}+1)\right\}-\mathrm{A}_{1}^{*}(\mathrm{n} \\
& -1) \mathrm{A}_{2}(\mathrm{n}) \mathrm{A}_{2}(\mathrm{n}-1)-\mathrm{A}_{1}^{*}(\mathrm{n} \\
& \left.+1) \mathrm{~A}_{2}(\mathrm{n}) \mathrm{A}_{2}(\mathrm{n}+1)\right]
\end{aligned}
$$

(Where $\chi=\frac{6 \mathrm{~K}_{4}}{\mathrm{~K}_{2}}$, The forbidden gap corresponds in the linear spectrum of the parameter $-\frac{\Delta}{\sigma}$ from 1 to -1 )

We can solve these equations for localized modes for narrow band where masses $\mathrm{M}$ and $\mathrm{m}$ are close to one another i.e. $\mathrm{m} / \mathrm{M}$ is of the order of unity .

\section{Volume 5 Issue 6, June 2016 www.ijsr.net}




\section{International Journal of Science and Research (IJSR)}

ISSN (Online): 2319-7064

Index Copernicus Value (2013): 6.14 | Impact Factor (2015): 6.391

\section{Solution for the Narrow Band}

The Parameters $\Delta, \sigma$ and $\chi$ are infinitesimals of the order $\epsilon$. So, we can use the expansion

$$
A_{j}(n \pm 1)=A_{j}(n) \pm \frac{d A_{j(n)}}{d n}+\epsilon^{2} \frac{d^{2} A_{j(n)}}{d n^{2}}-
$$

$\mathrm{j}=1,2 \ldots \ldots \ldots$

From equation $(3-\mathrm{a}, \mathrm{b})$ and the second order of $\in$, we assume $x=n$ and distance between atoms are equal . Substituting equation (4) in equation( 3 -a,b), we obtain

$$
\begin{gathered}
\frac{\mathrm{idA} A_{1}}{\mathrm{dx}}+\Delta \mathrm{A}_{1}-\sigma \mathrm{A}_{2}-\chi\left(\mathrm{A}_{1}\left|\mathrm{~A}_{1}\right|^{2}+2 \mathrm{~A}_{1}\left|\mathrm{~A}_{2}\right|^{2}-\mathrm{A}_{2}^{2} \mathrm{~A}_{1}^{*}\right) \\
\quad=0 \\
\frac{\mathrm{idA_{2 }}}{\mathrm{dx}}+\Delta \mathrm{A}_{2}-\sigma \mathrm{A}_{1}-\chi\left(\mathrm{A}_{2}\left|\mathrm{~A}_{2}\right|^{2}-2 \mathrm{~A}_{2}\left|\mathrm{~A}_{1}\right|^{2}-\mathrm{A}_{1}^{2} \mathrm{~A}_{1}^{*}\right)=
\end{gathered}
$$

Here $\mathrm{x}$ is treated as continuous co-ordinate. Since, we are interested in solutions that vanish in the limit $x \rightarrow \pm \infty, G=$ $0\left(\left|A_{1}\right|^{2}-\left|A_{2}\right|^{2}=G\right)$, and it is convenient to the represent the amplitudes of the forward and backward waves in the form

$$
A_{1}(x)=a(x) e^{-i \varphi(x)}, A_{2}(x)=a(x) e^{-i \varphi(x)}
$$

and

$$
A_{1}^{*}(x)=a(x) e^{-i \varphi(x)}, A_{2}^{*}(x)=a(x) e^{-i \varphi(x)}-(6)
$$

After solving equations (5) and(6) we get the equation for $\frac{d \varphi}{d x}$ and $\frac{\mathrm{da}}{\mathrm{dx}}$

$$
\begin{gathered}
\frac{\mathrm{d} \varphi}{\mathrm{dx}}=\Delta-\sigma \cos 2 \varphi+\chi \mathrm{a}^{2}[3-\cos 4 \varphi]- \\
\frac{\mathrm{da}}{\mathrm{dx}}=-\sigma \mathrm{a} \sin 2 \varphi-\chi \mathrm{a}^{3} \sin 4 \varphi-
\end{gathered}
$$

Using the integral of motion and condition $(\mathrm{G}=0)$ following from equation (7) and equation(8), we get

$$
\frac{\chi}{2} a^{4}(3-\cos 4 \varphi)+a^{2}(\Delta-\sigma \cos 2 \varphi)=0-
$$

Equation(9)can be expressed as the double Sine-Gordon equation of the phase $\varphi$

$$
\frac{d^{2} \varphi}{d x^{2}}-2 \Delta \sigma \sin 2 \varphi+\sigma^{2} \sin 4 \varphi=0-
$$

The soliton like solution corresponds to the path of the separatrix connecting the unstable saddle points. For $\Delta^{2}<$ $\sigma^{2}$, this corresponds to the frequency (i.e. $\sqrt{2} \omega_{1}<\omega<$ $\left.\sqrt{2} \omega_{2}\right)$ in the gap of linear spectrum. Solving equation (10) for phase we obtain two solutions

$$
\varphi_{\mathrm{I}}=\mathrm{m} \pi+\tan ^{-1}\left(\sqrt{\frac{\sigma-\Delta}{\sigma+\Delta}} \operatorname{coth} \sqrt{\sigma^{2}-\Delta^{2}}\left(\mathrm{x}-\mathrm{x}_{0}\right)\right)
$$

$$
\varphi_{\mathrm{II}}=(2 \mathrm{~m}+1) \frac{\pi}{2}-\tan ^{-1}\left(\sqrt{\frac{\sigma+\Delta}{\sigma-\Delta}} \operatorname{coth} \sqrt{\sigma^{2}-\Delta^{2}}\left(\mathrm{x}-\mathrm{x}_{0}\right)\right)-
$$

Substituting these solutions in the equation(7) we obtain the amplitude

$$
\mathrm{a}_{\mathrm{I}}^{2}=\frac{1}{\chi} \frac{\left\{(\sigma-\Delta) \operatorname{cosech}^{2} \sqrt{\sigma^{2}-\Delta^{2}}\left(\mathrm{x}-\mathrm{x}_{0}\right)\right\}\left\{1+\left(\frac{\sigma-\Delta}{\sigma+\Delta}\right) \operatorname{coth}^{2} \sqrt{\sigma^{2}-\Delta^{2}}\left(\mathrm{x}-\mathrm{x}_{0}\right)\right\}}{\left[1+\sigma\left(\frac{\sigma-\Delta}{\sigma+\Delta}\right) \operatorname{coth}^{2} \sqrt{\sigma^{2}-\Delta^{2}}\left(\mathrm{x}-\mathrm{x}_{0}\right)+\left(\frac{\sigma-\Delta}{\sigma+\Delta}\right)^{2} \operatorname{coth} \sqrt{\sigma^{2}-\Delta^{2}}\left(\mathrm{x}-\mathrm{x}_{0}\right)\right]}
$$

and

$$
\mathrm{a}_{\mathrm{II}}^{2}=\left[\frac{1}{\chi}\right] \frac{\left\{(\sigma+\Delta) \operatorname{cosech}^{2} \sqrt{\sigma^{2}-\Delta^{2}}\left(\mathrm{x}-\mathrm{x}_{0}\right)\right\}\left\{1+\left(\frac{\sigma+\Delta}{\sigma-\Delta}\right) \operatorname{coth}^{2} \sqrt{\sigma^{2}-\Delta^{2}}\left(\mathrm{x}-\mathrm{x}_{0}\right)\right\}}{\left[1+\sigma\left(\frac{\sigma+\Delta}{\sigma-\Delta}\right) \operatorname{coth}^{2} \sqrt{\sigma^{2}-\Delta^{2}}\left(\mathrm{x}-\mathrm{x}_{0}\right)+\left(\frac{\sigma+\Delta}{\sigma-\Delta}\right)^{2} \operatorname{coth}^{4} \sqrt{\sigma^{2}-\Delta^{2}}\left(\mathrm{x}-\mathrm{x}_{0}\right)\right]}
$$

Expression for $\mathrm{a}_{\mathrm{II}}^{2}$ as the same as $\mathrm{a}_{\mathrm{I}}^{2}$, when $\Delta$ replace by $(-\Delta)$, the $a_{I}^{2}$ correspond to case $\mathrm{x}<0$ and $a_{I I}^{2}$ correspond to case $\chi>0$, At the point $\omega=\omega^{*}, \Delta$ is equal to zero i.e. edge of the band, the equation (13) and equation (14) becomes same and expression is given by

$$
\mathrm{a}_{1}^{2}=\mathrm{a}_{\mathrm{II}}^{2}=\frac{\sigma}{\chi} \frac{\cosh 2 \sigma\left(\mathrm{x}-\mathrm{x}_{0}\right)}{\cosh 4 \sigma\left(\mathrm{x}-\mathrm{x}_{0}\right)}-
$$

A fundamental condition for the existence of localized modes in the spatial modulation of the phase, which gives the nonlinear optical switching of periodic chain. In a diatomic chain, the field in the forbidden gap is a standing wave. The location of maxima and minima of this wave depend on the frequency. For a linear chain with $\omega \approx$ $\sqrt{2} \omega_{1}(\sigma=\Delta)$ the maxima falls on the sites with the heavy atoms and the minima falls on the light (atoms with mass $\mathrm{m}$ are at rest). At $\omega \approx \sqrt{2} \omega_{2}(\sigma=-\Delta)$, the situation is the reverse only the light atoms vibrate. The action of the nonlinearity is most effective under these conditions. If $\omega \approx \sqrt{2} \omega_{1}$ then as the amplitude of the wave increases equation (1) implies that coupling co - efficient $\mathrm{K}_{2}$ and the natural frequency of vibration of the heavy particles increase $\left(\mathrm{K}_{4}<0\right)$. If the natural frequency of the light particles remains the same the forbidden gap become wider. Near $\omega \approx \omega^{*}$, both natural frequencies are lower, so the width of the forbidden gap is unchanged. The phase modulation in the region of excitation given by equations (11) and(12) provides exactly the conditions under which the necessary shift in the maxima of the standing wave would correspond to the sites of either the heavy $(\chi<0)$ or the light $(\chi>0)$ particles. Outside the region the standing wave acquires form characteristics for a linear chain.

Using equations(11) and(13) the amplitude of the vibration of the heavy atoms $\mathrm{A}$ and the light atoms B for $\chi<0$ can be written as

For heavy atoms (A)

and

$$
\mathrm{A}=\mathrm{A}_{1}+\mathrm{A}_{2}=2 \mathrm{a} \cos \varphi-
$$

For light atoms (B)

$$
B=A_{1}-A_{2}=2 i \sin \varphi
$$

\section{Volume 5 Issue 6, June 2016 www.ijsr.net}




\section{International Journal of Science and Research (IJSR) \\ ISSN (Online): 2319-7064}

Index Copernicus Value (2013): 6.14 | Impact Factor (2015): 6.391

When $\Delta$ is equal to zero from equations(16)and (17) we obtain

$$
A=2 \sqrt{\frac{\sigma}{\chi}} \frac{\sinh \sigma\left(x-x_{0}\right)}{\sqrt{\cosh 4 \sigma\left(x-x_{0}\right)}}-
$$

and

$$
\mathrm{B}=2 \sqrt{\frac{\sigma}{\chi}} \frac{\cosh \sigma\left(\mathrm{x}-\mathrm{x}_{0}\right)}{\sqrt{\cosh 4 \sigma\left(\mathrm{x}-\mathrm{x}_{0}\right)}}-
$$

When $\Delta$ is not equal to zero, form equation(16) and (17) we obtain

$$
\frac{2}{\sqrt{|\chi|}} \frac{\sqrt{\sigma(1-\mathrm{N})} \operatorname{cosec} \mathrm{h} \sigma \sqrt{1-\mathrm{N}^{2}}\left(\mathrm{x}-\mathrm{x}_{0}\right)}{\left\{1+6\left(\frac{1-\mathrm{N}}{1+\mathrm{N}}\right) \operatorname{coth}^{2} \sigma \sqrt{1-\mathrm{N}^{2}}\left(\mathrm{x}-\mathrm{x}_{0}\right)+\left(\frac{1-\mathrm{N}}{1+\mathrm{N}}\right)^{2} \operatorname{coth}^{4} \sigma \sqrt{1-\mathrm{N}^{2}}\left(\mathrm{x}-\mathrm{x}_{0}\right)\right\}^{\frac{1}{2}}}
$$

$$
\frac{2}{\sqrt{\chi}} \frac{\left\{\sqrt{\sigma(1-\mathrm{N})} \operatorname{Cosech} \sigma \sqrt{1-\mathrm{N}^{2}}\left(\mathrm{x}-\mathrm{x}_{0}\right)\right\}\left\{\sqrt{\frac{1-\mathrm{N}}{1+\mathrm{N}} \operatorname{coth} \sigma \sqrt{1-\mathrm{N}^{2}}\left(\mathrm{x}-\mathrm{x}_{0}\right)}\right\}}{\left\{1+6\left(\frac{1-\mathrm{N}}{1+\mathrm{N}}\right) \operatorname{coth}^{2} \sigma \sqrt{1-\mathrm{N}^{2}}\left(\mathrm{x}-\mathrm{x}_{0}\right)+\left(\frac{1-\mathrm{N}}{1+\mathrm{N}}\right)^{2} \operatorname{coth}^{4} \sigma \sqrt{1-\mathrm{N}^{2}}\left(\mathrm{x}-\mathrm{x}_{0}\right)\right\}^{\frac{1}{2}}}
$$

For $\chi>0$, from equations (16) and (17), when $\Delta$ is equals to zero

and

$$
\mathrm{A}=2 \sqrt{\frac{\sigma}{\chi}} \frac{\cosh \sigma\left(\mathrm{x}-\mathrm{x}_{0}\right)}{\sqrt{\cosh 4 \sigma\left(\mathrm{x}-\mathrm{x}_{0}\right)}}-
$$

$$
\mathrm{B}=2 \sqrt{\frac{\sigma}{\chi}} \frac{\sinh \sigma\left(\mathrm{x}-\mathrm{x}_{0}\right)}{\sqrt{\cosh 4 \sigma\left(\mathrm{x}-\mathrm{x}_{0}\right)}}-
$$

When $\Delta$ is not equal to zero, from equations (16) and (17) we obtain

$$
\frac{2}{\sqrt{|\chi|}} \frac{\left\{\sqrt{\sigma(1+N)} \operatorname{cosec} h \sigma \sqrt{1-N^{2}}\left(x-x_{0}\right)\right\}\left\{\sqrt{\frac{1+N}{1-N} \operatorname{coth} \sigma \sqrt{1-N^{2}}\left(x-x_{0}\right)}\right\}}{\left\{1+6\left(\frac{1+N}{1-N}\right) \operatorname{coth}^{2} \sigma \sqrt{1-\mathrm{N}^{2}}\left(x-x_{0}\right)+\left(\frac{1+N}{1-N}\right)^{2} \operatorname{coth}^{4} \sigma \sqrt{1-\mathrm{N}^{2}}\left(x-x_{0}\right)\right\}^{\frac{1}{2}}}
$$

$$
\frac{2}{\sqrt{|\chi|}} \frac{\sqrt{\sigma(1+\mathrm{N})} \operatorname{cosech} \sigma \sqrt{1-\mathrm{N}^{2}}\left(\mathrm{x}-\mathrm{x}_{0}\right)}{\left\{1+6\left(\frac{1+\mathrm{N}}{1-\mathrm{N}}\right) \operatorname{coth}^{2} \sigma \sqrt{1-\mathrm{N}^{2}}\left(\mathrm{x}-\mathrm{x}_{0}\right)+\left(\frac{1+\mathrm{N}}{1-\mathrm{N}}\right)^{2} \operatorname{coth}^{4} \sigma \sqrt{1-\mathrm{N}^{2}}\left(\mathrm{x}-\mathrm{x}_{0}\right)\right\}^{\frac{1^{2}}{2}}}
$$

The amplitudes of the heavy atom (A) and the light atom (B) with position are shown in figures from 1 to 4 for different cases.

$$
\mathrm{A},\left(\frac{\mathrm{m}}{\mathrm{M}}\right)=0.9, \frac{\omega^{2}}{\omega_{1}^{2}}=2.0, \mathrm{~A}=\left[\frac{0.3162 \operatorname{Csch}[0.044 x]}{\sqrt{1+0.1041 \operatorname{Coth}[0.044 x]^{4}+1.9355 \operatorname{Coth}[0.044 x]^{2}}},\{x,-100,100\}\right]
$$

\section{Chi Greater than Zero}

$\mathrm{A},\left(\frac{\mathrm{m}}{\mathrm{M}}\right)=0.9, \frac{\omega^{2}}{\omega_{1}^{2}}=2.05 \mathrm{~A}$

$=\left[\frac{0.56 \operatorname{Csch}[0.044 x] 1.7607 \operatorname{Coth}[0.044 x]}{\sqrt{1+9.61 \operatorname{Coth}[0.044 x]^{4}+18.6 \operatorname{Coth}[0.044 x]^{2}}},\{x,-80,80\}\right]$

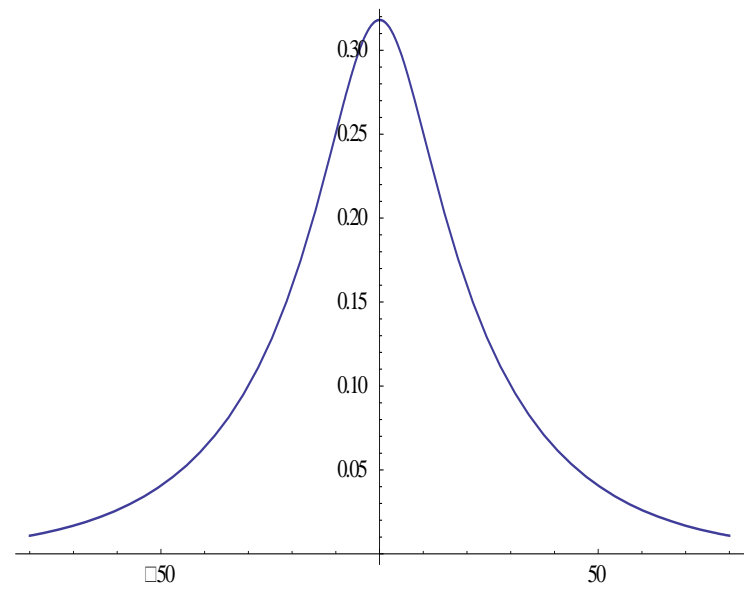

Figure 1: Amplitude (A) v/s Displacement (x)

$\mathrm{B},\left(\frac{\mathrm{m}}{\mathrm{M}}\right)=0.9, \frac{\omega^{2}}{\omega_{1}^{2}}=2 . \mathrm{B}$

$=\left[\frac{0.56 \operatorname{Csch}[0.044 x]}{\sqrt{1+9.61 \operatorname{Coth}[0.044 x]^{4}+18.6 \operatorname{Coth}[0.044 x]^{2}}},\{x,-80,80\}\right]$

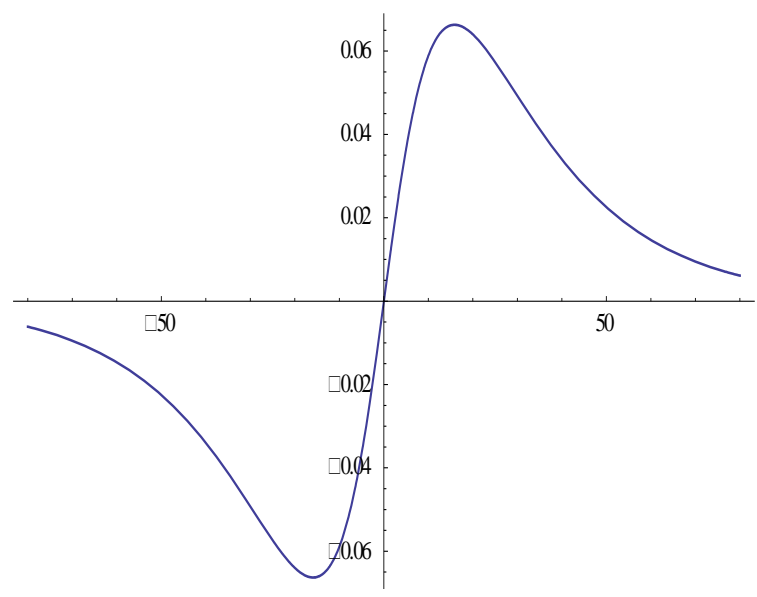

Figure 2: Amplitude (B) v/s Displacement (x)

\section{Chi Less Than Zero}

\section{Volume 5 Issue 6, June 2016 www.ijsr.net}

Licensed Under Creative Commons Attribution CC BY 


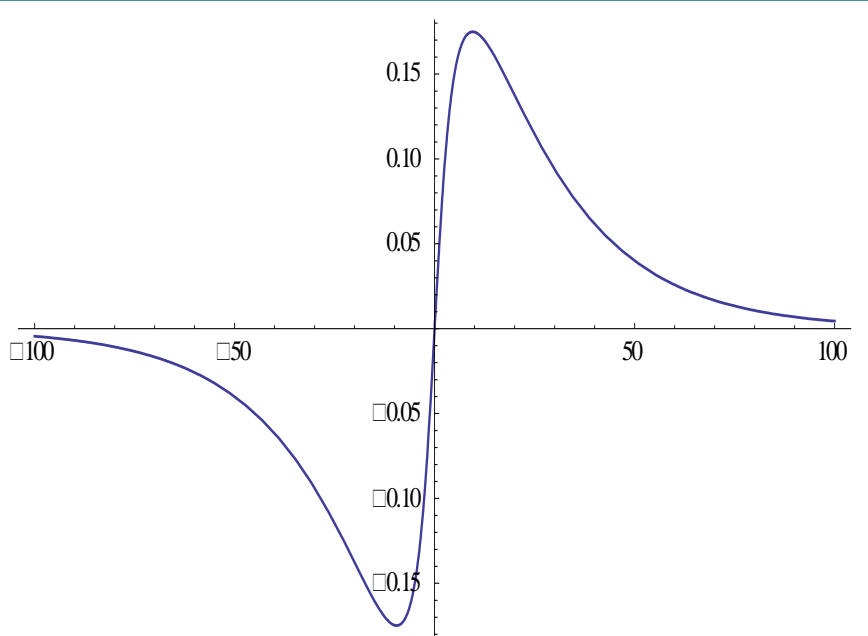

Figure 3: Amplitude (A) v/s Displacement (x)

$$
\mathrm{B},\left(\frac{\mathrm{m}}{\mathrm{M}}\right)=0.9, \frac{\omega^{2}}{\omega_{1}^{2}}=2.05 \mathrm{~B}=\left[\frac{0.3162 \operatorname{Csch}[0.044 x] 0.568 \operatorname{Coth}[0.044 x]}{\sqrt{1+0.1041 \operatorname{Coth}[0.044 x]^{4}+1.9355 \operatorname{Coth}[0.044 x]^{2}}},\{x,-50,50\}\right]
$$

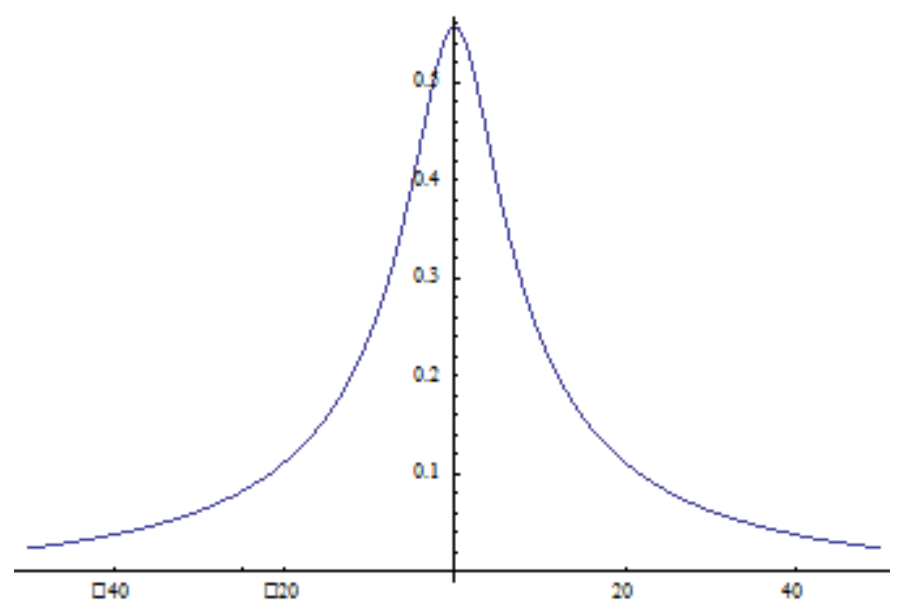

Figure 4: Amplitude (B) v/s Displacement (x)

\section{Concluding Remarks}

The solutions we have obtained are different from those reported earlier in literature[13,14]. These correspond to the region where the corresponding linear lattice is transparent. The amplitude of the heavy and light atoms of diatomic chains is calculate for three frequencies (one near the lower edge of the band gap, the other near the upper edge of the band gap ). The existence of these localized modes is because of the spatial phase modulation. Structurally the modes found are close to the even and odd modes of the monoatomic chain [15]. In the present case presence of atoms of different kinds results in a unique combination of these modes with the same sign of the nonlinearity.

For the case of the wide band gap (arbitrary ratio $\mathrm{m} / \mathrm{M}$ ) diatomic chain the solutions have been reported in literature [16 ] near the edges of the band gap (detuning of the excitation frequency). Therefore it will be of interest to investigate the existence of such solutions for the entire frequency spectrum in the band gap.

\section{References}

[1] M.Akoi,S.Takeno and A.SieversJ.Phys. Soc. Jpn.62,4295(1993)

[2] M.Akoi, J.Phys. Soc. Jpn.61,3024(1992)

[3] Bambi Hu,Guoxing Huang Manuel.G.Velarde(2 issue), Phy.Rev.E62,2827-2839(2000)

[4] A.Yariv and P. Yeh, Optical waves in crystals, Wiley N.Y.(1984)

[5] C. Elaichi,Porc. IRE64,22(1976)

[6] S. Y. Karpov and S. N. Stolyarov.Usp. Fi2 Nauk 163,63(1993)

[7] O.L. Milla and S.E. Trullinger Phys. Rev. B36,947(1987)

[8] A.B. Aceves and S. Wabnitz, Phys. Lett. A141,37(1989)

[9] V.Hizhnyakov, A. Shelkan, M. Klopov, S.A. Kieselev, and A.J. Sievers, Phy. Rev. B73,224-302(2006)

[10]O. N. Emakova, V.F. Marchenko, and A.P. Sukhorukov, J.Phys. Solid State 39(1)136 - 139(1997)

[11]O.A. Chubykal, S.A. Kovalev and O.V. Usaternko, Phys. Rev. B47,3153(1993)

[12] W. Chen and D. L. Mills, Phys. Rev. Lett. 58, 169 (1987)

[13] O. N. Emakova, V.F. Marchenko, and A.P. Sukhorukov, Fiz.Tveid. Tela(St. Petersburg) 39,158162(Jan.1997)

[14]A S Gorshkov, O N Ermakova and V F Marchenko, Nonlinearity 10 (1997) 1007-1014

[15] S. A. Kovalev, O. V. Usashenko, and O. A. Chubykalo, Fiz. Tverd.Tela (St. Petersburg) 35, 693 (1993) [Phys. Solid State 35, 356 (1993)]

[16]O. N. Emakova, V.F. Marchenko, and A.P. Sukhorukov, Fiz. Tverd.Tela (St. Petersburg) 39,158 162(1997) 